\title{
Three-Way Effect of Cyanine Dye on the Structure and Function of Mitochondria
}

\author{
Kikuji Yamashita, ${ }^{a}$ Tomokazu Ichikawa, ${ }^{b, c}$ Takenori Yamamoto, ${ }^{b, c}$ Masatoshi Kataoka, ${ }^{a, b}$ \\ Yoshinori Nakagawa, ${ }^{d}$ Hiroshi Terada, ${ }^{e}$ and Yasuo Shinohara ${ }^{*, b, c, f}$
}

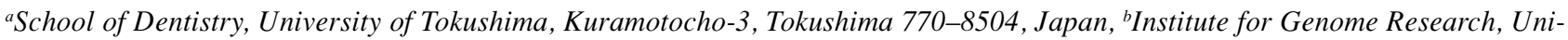
versity of Tokushima, Kuramotocho-3, Tokushima 770-8503, Japan, 'Faculty of Pharmaceutical Sciences, University of Tokushima, Shomachi-1, Tokushima 770-8505, Japan, 'Drugs and Cosmetics Research Center, Hayashibara Biochemical Laboratories, Inc., Shimoishii-1, Okayama 700-0907, Japan, ${ }^{e}$ Faculty of Pharmaceutical Sciences, Tokyo University of Science, Ichigaya, Shinjuku-ku, Tokyo 162-0826, Japan, and ${ }^{f}$ Single-Molecule Bioanalysis Laboratory, National Institute of Advanced Industrial Science and Technology (AIST), Hayashicho 2217-14, Takamatsu 761-0395, Japan

(Received May 23, 2003; Accepted August 4, 2003)

\begin{abstract}
Previously, we found that the cationic cyanine dye tri-S- $\mathrm{C}_{4}(5)$ uncoupled mitochondrial oxidative phosphorylation by acting as an inducer of the mitochondrial permeability transition (PT). In the present study, the actions of cyanine dyes such as tri-S- $\mathrm{C}_{4}(5)$ and tri-S-C $\mathrm{C}_{7}(5)$ on the mitochondrial structures and functions were further characterized. In the presence of inorganic phosphate $(\mathrm{Pi})$, cyanine dyes were found to accelerate mitochondrial oxygen consumption that was partially sensitive to the PT inhibitor cyclosporin A (CsA). However, not only the CsAsensitive but also CsA-insensitive acceleration of mitochondrial respiration was induced by cyanine dyes; and both of them were found to be associated with the release of mitochondrial cytochrome c. On the contrary, in the absence of $\mathrm{Pi}$, moderate acceleration of respiration was induced by cyanine dyes, but this respiratory effect was not associated with the induction of swelling or the release of mitochondrial cytochrome $\mathrm{c}$. Thus, cyanine dyes were concluded to have 3 different effects on the mitochondrial functions depending on the Pi status.
\end{abstract}

Key words — mitochondria, permeability transition, cyanine dyes, release of cytochrome c, apoptosis

\section{INTRODUCTION}

Cyanine dyes with a positive charge(s) have been commonly used for estimating the membrane potential across the inner mitochondrial membrane. ${ }^{1,2)}$ However, at higher concentrations than those used for monitoring the membrane potential, some of them were found to act as uncouplers of mitochondrial oxidative phosphorylation. ${ }^{3-6)}$ Unlike the weakly acidic uncouplers such as FCCP and SF6847, ${ }^{7-10)}$ the uncoupling action of cyanine dyes requires inorganic phosphate $(\mathrm{Pi})$ and is accompanied by mitochondrial swelling. These features are very similar to those induced by $\mathrm{Ca}^{2+}$. Recent studies on $\mathrm{Ca}^{2+}$-induced "uncoupling" show that $\mathrm{Ca}^{2+}$ increases the permeability of the inner mitochondrial membrane to various solutes by forming permeability transition (PT) pores, ${ }^{11-13)}$ although the

*To whom correspondence should be addressed: Institute for Genome Research, University of Tokushima, Kuramotocho-3, Tokushima 770-8504, Japan. Tel.: +81-88-633-9145; Fax: +8188-633-9146; E-mail: yshinoha@genome.tokushima-u.ac.jp molecular features of these pores are still unclear. For characterization of the PT pore, characterization of the PT induced by various reagents, as well as genetic approaches, would seem to be helpful.

Recently, we obtained data suggesting that cationic uncouplers such as the cyanine dye 2,2'-[3-[2(3-butyl-4-methyl-2-thiazolin-2-ylidene) ethylidene] propenylene]-bis[3-butyl-4-methyl thiazolinium iodide] [tri-S- $\left.\mathrm{C}_{4}(5)\right]$ and copper- $O$-phenanthroline induced the PT pore in the inner mitochondrial membrane. ${ }^{14)}$ As an extension of our previous study, in this study, we examined the actions of cyanine dyes on mitochondrial structure and function in more detail. Namely, we studied the difference between the actions of cyanine dyes and $\mathrm{Ca}^{2+}$ on mitochondrial respiration in terms of the sensitivity to the PT inhibitor cyclosporin A (CsA), the differences in Pi dependence of actions of cyanine dyes and $\mathrm{Ca}^{2+}$, and features of the release of cytochrome $\mathrm{c}$ induced by cyanine dyes. Our results clearly showed that the accelerated mitochondrial oxygen consumption induced by cyanine dyes in cooperation with Pi was only partially sensitive to CsA and that both CsA- 
sensitive and insensitive actions were effective for the release of mitochondrial cytochrome c. Furthermore, cyanine dyes were found to cause moderate acceleration of mitochondrial respiration in the absence of Pi that was not associated with cytochrome $c$ release.

\section{MATERIALS AND METHODS}

Materials _ CsA was kindly donated by Novartis Pharma K.K. (Tokyo). An enhanced chemiluminescence (ECL) immunodetection kit was purchased from Amersham Pharmacia Biotech. Cyanine dyes of tri-S- $\mathrm{C}_{4}(5)$ and 2,2'-[3-[2-(3-heptyl-4-methyl-2thiazolin-2-ylidene) ethylidene] propenylene]-bis[3heptyl-4-methyl thiazolinium iodide] [tri-S- $\mathrm{C}_{7}(5)$ ] are product of Hayashibara Biochemical Laboratories, Inc.

Isolation of Mitochondria and Measurement of Mitochondrial Respiration - Mitochondria were isolated from the liver of male Wistar rats according to the procedure described previously. ${ }^{3-5)} \mathrm{Mi}$ tochondrial respiration was measured with a Clark oxygen electrode (Yellow Springs, model 5331) at $25^{\circ} \mathrm{C}$. For this, mitochondria were first suspended in $+\mathrm{Pi}$ medium $(200 \mathrm{mM}$ sucrose and $10 \mathrm{mM}$ potassium phosphate buffer, $\mathrm{pH}$ 7.4) or $-\mathrm{Pi}$ medium (200 mM sucrose, $10 \mathrm{mM} \mathrm{KCl}$, and $10 \mathrm{mM}$ Tris-Cl buffer; $\mathrm{pH}$ 7.4) to make a final concentration of $0.7 \mathrm{mg}$ protein $/ \mathrm{ml}$. Then, they were energized with $10 \mathrm{mM}$ succinate (plus $0.5 \mu \mathrm{g} / \mathrm{ml}$ rotenone). To examine the effects of cyanine dyes, we added these dyes in dimethylsulfoxide (DMSO) solution to the energized mitochondria. Addition of DMSO itself was also performed as a negative control (data not shown). For the measurement of mitochondrial swelling, turbidity of mitochondrial suspension at $700 \mathrm{~nm}$ was monitored in a dual-wavelength spectrophotometer, a Shimadzu UV-3000.

Measurement of Release of Mitochondrial Cytochrome c - To measure the release of cytochrome c from mitochondria, we treated an aliquot $(100 \mu \mathrm{l})$ of mitochondrial suspension with a given cyanine dye or not for $4 \mathrm{~min}$, placed it in an Eppendorf centrifuge tube, promptly centrifuged it, and then separated the supernatant from the precipitant. The precipitated mitochondria were resuspended in $100 \mu \mathrm{l}$ of the incubation medium, and $9 \mu \mathrm{l}$ of this suspension $(\mathrm{P})$ and $15 \mu \mathrm{l}$ of supernatant $(\mathrm{S})$ were individually subjected to sodium dodecyl sulphate polyacrylamide gel electrophoresis (SDS-
PAGE). Cytochrome $\mathrm{c}$ retained in mitochondria or released into the incubation medium was detected with a specific antibody against cytochrome $\mathrm{c}$, which had been previously prepared by using a synthetic peptide with the amino acid sequence of HTVEKGGKHKTGPNLHGLFC as an immunogen.

\section{RESULTS}

\section{In the Presence of Pi, Cyanine Dyes Accelerated Mitochondrial Respiration in both CsA-Sensitive and Insensitive Manners}

In the previous study, we found that cyanine dyes such as tri-S- $\mathrm{C}_{4}(5)$ caused acceleration of mitochondrial respiration and mitochondrial swelling when $\mathrm{Pi}$ was present in the incubation medium. ${ }^{5)}$ Furthermore, these changes induced by tri-S- $\mathrm{C}_{4}(5)$ were markedly reduced by the addition of $\mathrm{CsA},{ }^{14)}$ which is known to be a specific inhibitor of mitochondrial PT. ${ }^{15,16)}$ However, recent studies indicated that in addition to the "ordinary" CsA-sensitive PT as that induced by $\mathrm{Ca}^{2+}$, a PT insensitive to CsA was induced by several inducers such as signal peptide and butylated hydroxytoluene. ${ }^{17,18)}$ However, it is not clear whether the PT induced by cyanine dye is completely sensitive to CsA. Thus, we first examined the effect of CsA on the action of tri-S- $\mathrm{C}_{4}(5)$ with respect to mitochondrial functions in a quantitative manner. When induction of mitochondrial PT is assessed, turbidities of mitochondrial suspension, reflecting their volume change, are often measured. However, as reported recently, ${ }^{19)}$ mitochondrial volume change could be happen even under the condition at which PT is not occurred, and is not so sensitive for detecting the changes in membrane status. On the contrary, rates of mitochondrial oxygen consumption severely reflect the status of inner mitochondrial membrane. Thus, in the present study, we mainly measured rates of oxygen consumption of mitochondria treated with various reagents. Furthermore, we also examined the effect of another cyanine dye, tri-S- $\mathrm{C}_{7}(5)$, having a saturated hydrocarbon side chain $(n=7)$ longer than that of tri-S- $\mathrm{C}_{4}(5)$ $(n=4)$, because we reported earlier that tri-S- $\mathrm{C}_{7}(5)$ showed similar actions toward mitochondrial functions as tri-S- $\mathrm{C}_{4}(5) .{ }^{3,4)}$ For a control, the effect of CsA on PT induced by $\mathrm{Ca}^{2+}$ was also examined.

As reported previously, in the presence of $\mathrm{Pi}$, cyanine dyes tri-S- $\mathrm{C}_{4}(5)$ and tri-S- $\mathrm{C}_{7}(5)$ caused remarkable acceleration of mitochondrial respiration that was accompanied by mitochondrial swelling. ${ }^{4,5)}$ 
These effects of cyanine dyes on mitochondria were very similar to those of $\mathrm{Ca}^{2+}$. However, unlike the respiration induced by $\mathrm{Ca}^{2+}$, which was completely inhibited by $\mathrm{CsA}$ over a wide range of $\mathrm{Ca}^{2+}$ concentration, that induced by tri-S- $\mathrm{C}_{4}(5)$ and tri-S- $\mathrm{C}_{7}(5)$ was only partially sensitive to CsA (Figs. 1 and 2). Namely, when mitochondria were treated with low concentrations of cyanine dyes [less than $50 \mu \mathrm{M}$ for tri-S- $\mathrm{C}_{4}(5)$ and $2 \mu \mathrm{M}$ for tri-S- $\left.\mathrm{C}_{7}(5)\right]$, the accelerated respiration was remarkably reduced by CsA. However, when mitochondria were treated with cyanine dyes at higher concentrations, accelerated respiration was not inhibited by CsA, even CsA had the high concentration of $10 \mu \mathrm{M}$ was utilized (data not shown). Thus, cyanine dyes were concluded to accelerate mitochondrial respiration in both CsA-sensitive and CsA-insensitive manners.

\section{In the Absence of Pi, Cyanine Dyes at Higher Con- centrations Caused Moderate Acceleration of Mi- tochondrial Respiration Insensitive to CsA}

When the effects of cyanine dyes on mitochondrial respiration were compared with those of $\mathrm{Ca}^{2+}$, a remarkable difference was observed in the absence of Pi. Namely, in the absence of $\mathrm{Pi}, \mathrm{Ca}^{2+}$ was completely ineffective in stimulating the mitochondrial respiration even at high concentrations such as $500 \mu \mathrm{M}$. However, the cyanine dyes stimulated the mitochondrial respiration at higher concentrations, although their actions were much more moderate than those observed in the presence of Pi (Figs. 1 and 2). Furthermore, the accelerating effects of cyanine dyes on mitochondrial respiration without $\mathrm{Pi}$ were almost completely insensitive to CsA (Fig. 2). Thus, cyanine dyes were concluded to have 3 different effects on mitochondria: in the presence of $\mathrm{Pi}$, they accelerated mitochondrial respiration in both CsA-sensitive and CsA-insensitive manners, and in the absence of $\mathrm{Pi}$, they caused a moderate acceleration of mitochondrial respiration in a CsA-insensitive manner.

\section{In the Presence of Pi, Mitochondrial Cytochrome c Could be Released by Cyanine Dyes}

Upon the induction of the mitochondrial PT, the release of cytochrome $c$ is known to occur. ${ }^{20-22)}$ Since released cytochrome $\mathrm{c}$ was shown to trigger the subsequent steps of programmed cell death, called apoptosis, ${ }^{20-22)}$ we found it interesting to examine whether the release of mitochondrial cytochrome $\mathrm{c}$ would be induced by the cyanine dyes.

As shown in Fig. 3, when the PT was induced

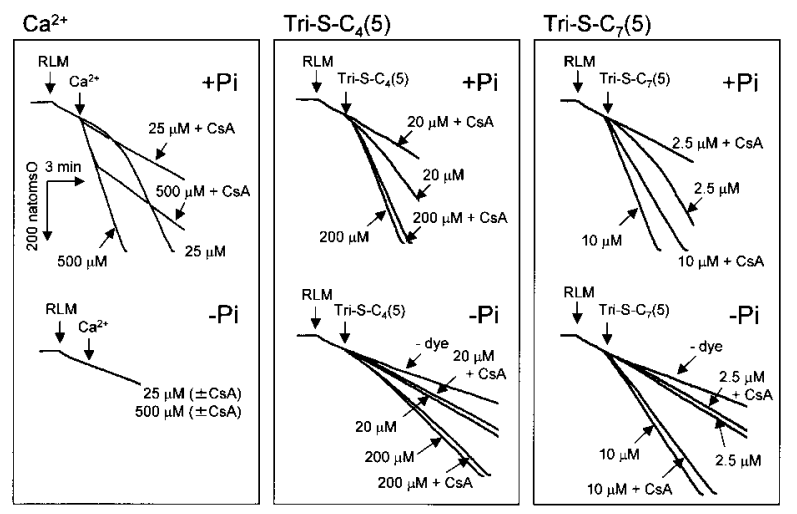

Fig. 1. Stimulatory Effects of $\mathrm{Ca}^{2+}$ and Cyanine Dyes on Mitochondrial Respiration and Sensitivity of These Effects to CsA

Mitochondrial respiration observed in the presence of $\mathrm{Ca}^{2+}$, tri-S$\mathrm{C}_{4}(5)$ or tri-S- $\mathrm{C}_{7}(5)$ was measured. For this, mitochondria were suspended in $+\mathrm{Pi}$ or $-\mathrm{Pi}$ medium to make final protein concentrations of $0.7 \mathrm{mg} /$ $\mathrm{ml}$, energized by the addition of $10 \mathrm{mM}$ succinate $(+0.5 \mu \mathrm{g} / \mathrm{ml}$ rotenone), and treated with various reagents. Upper and lower traces represent the results observed in the presence and absence of $\mathrm{Pi}$, respectively. In the case of $+\mathrm{CsA}$, it was added to make a final concentration of $1 \mu \mathrm{M}$. RLM, rat liver mitochondria.
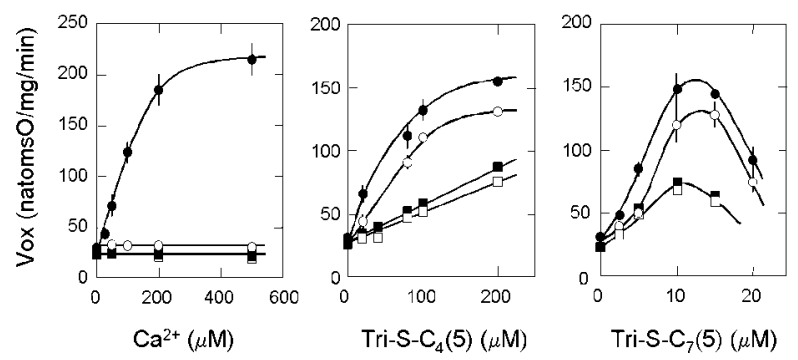

Fig. 2. Dose Response Curve of the Effect of $\mathrm{Ca}^{2+}$ and Cyanine Dyes on Mitochondrial Respiration

Experimental conditions were as described for Fig. 1, and rates of respiration were determined from the slopes of oxygen traces at $2 \mathrm{~min}$ or 4 min after addition $\mathrm{Ca}^{2+}$ or cyanine dyes, respectively. Circles and squares represent the results observed with +Pi medium and -Pi medium, respectively; and closed and open symbols, represent those obtained in the absence and presence of $1 \mu \mathrm{M} \mathrm{CsA}$, respectively.

by $500 \mu \mathrm{M} \mathrm{Ca}^{2+}$, a significant amount of cytochrome $\mathrm{c}$ was released from the mitochondria, representing approx. 35\% of the total cytochrome $\mathrm{c}$ in mitochondria; and this release was completely prevented by the addition of CsA. On the contrary, the release of mitochondrial cytochrome $c$ induced by cyanine dyes was partially sensitive for CsA. Namely, as also shown in Fig. 3, when mitochondria were treated with relatively low concentrations of cyanine dyes in the presence of $\mathrm{Pi}$, the release of mitochondrial cytochrome $\mathrm{c}$ was not significant but that amount was almost completely prevented by the addition of CsA. However, when mitochondria were treated with 


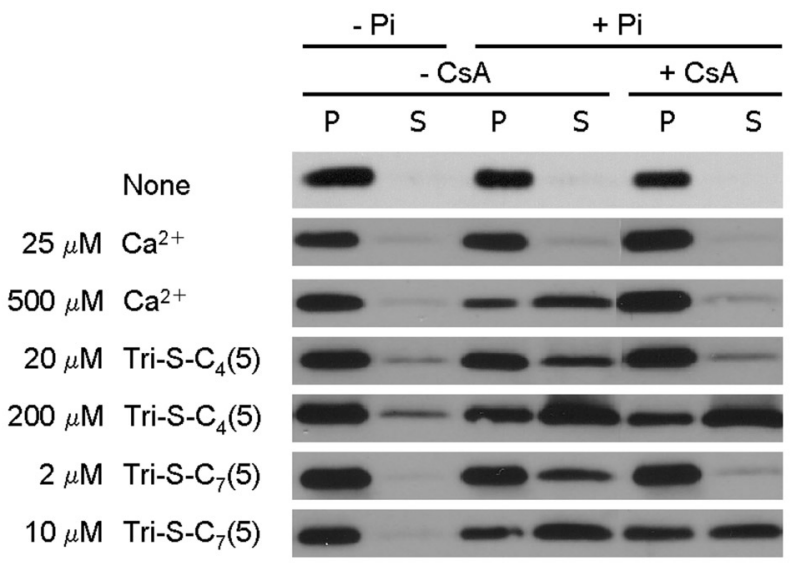

Fig. 3. Release of Mitochondrial Cytochrome $\mathrm{c}$ Induced by $\mathrm{Ca}^{2+}$ and Cyanine Dyes and Sensitivity of the Release to CsA

Mitochondria were treated with $\mathrm{Ca}^{2+}$, tri-S- $\mathrm{C}_{4}(5)$ or tri-S- $\mathrm{C}_{7}(5)$ under various conditions. After $2 \min \left(\mathrm{Ca}^{2+}\right)$ or $4 \mathrm{~min}$ (cyanine dyes) of incubation, the mitochondria were separated from the supernatant by centrifugation. Proteins involved in supernatant $(\mathrm{S})$ and precipitant $(\mathrm{P})$ were subjected to SDS-PAGE, and then the content of cytochrome $\mathrm{c}$ in each was assessed by using anti-cytochrome $\mathrm{c}$ antibody.

higher concentrations of cyanine dyes with $\mathrm{Pi}$, the release of mitochondrial cytochrome $\mathrm{c}$ was more significant and was not completely prevented by CsA. These results well agreed with the results on stimulation of respiration showing that the release of cytochrome $\mathrm{c}$ was well dependent on the acceleration of respiration.

In the Absence of Pi, Cyanine Dyes Neither Induce the Release of Mitochondrial Cytochrome $c$ Nor Mitochondrial Swelling

As described above, both cyanine dyes caused a slight acceleration of mitochondrial respiration in the absence of Pi. It should be noted that the rates of respiration induced by higher concentrations of cyanine dyes [such as $200 \mu \mathrm{M}$ tri-S- $\mathrm{C}_{4}(5)$ and $10 \mu \mathrm{M}$ tri-S- $\left.\mathrm{C}_{7}(5)\right]$ in the absence of Pi were larger than those induced by lower concentrations of them [such as $20 \mu \mathrm{M}$ tri- $-\mathrm{S}_{4}(5)$ and $2 \mu \mathrm{M}$ tri-S- $\left.\mathrm{C}_{7}(5)\right]$ in the presence of $\mathrm{Pi}$ (Fig. 2). If the degree of cytochrome c release shows parallel relationship just with rate of respiration, cytochrome $\mathrm{c}$ should be also released when mitochondria were treated with higher concentration of cyanine dye in the absence of Pi. However, the release of cytochrome $\mathrm{c}$ was observed only under the latter conditions and was almost negligible in the absence of Pi (Fig. 3).

As stated above, in the presence of $\mathrm{Pi}$, remarkable mitochondrial swelling accompanied by accelerated respiration was caused by the cyanine dyes ${ }^{5,14}$
A

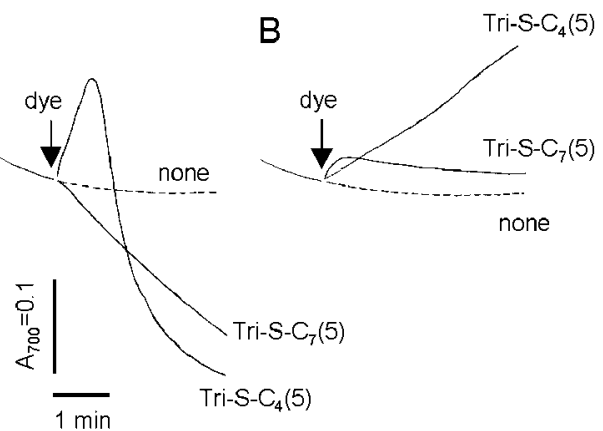

Fig. 4. Cyanine Dyes Induce Mitochondrial Swelling only in the Presence of Pi

Mitochondria were suspended in $+\mathrm{Pi}(\mathrm{A})$ or $-\mathrm{Pi}$ medium (B) to make final protein concentrations of $0.7 \mathrm{mg} / \mathrm{ml}$, energized by the addition of $10 \mathrm{mM}$ succinate $(+0.5 \mu \mathrm{g} / \mathrm{ml}$ rotenone $)$, and treated with $200 \mu \mathrm{M}$ tri$\mathrm{S}_{-} \mathrm{C}_{4}(5)$ or $10 \mu \mathrm{M}$ tri-S- $\mathrm{C}_{7}(5)$. Changes in mitochondrial volume were monitored by measuring the turbidity of the mitochondrial suspension at $700 \mathrm{~nm}$. Broken traces represent the results obtained without treatment with cyanine dyes.

(See also Fig. 4A). However, in the absence of Pi, swelling was not induced even when mitochondria were treated with higher concentrations of the dyes (Fig. 4B). It is noteworthy that, in the absence of $\mathrm{Pi}$, the turbidity of the mitochondrial suspension treated with tri-S $-\mathrm{C}_{4}(5)$ rather increased gradually. The meaning of this increase is still uncertain; however, we clearly showed that mitochondrial swelling was not induced by cyanine dyes in the absence of Pi.

Based on these results, the acceleration of mitochondrial respiration in the absence of Pi by cyanine dyes seems to have occurred by a mechanism different from that causing the PT, and therefore, not associated with cytochrome $\mathrm{c}$ release.

\section{DISCUSSION}

In the previous study, we showed that the action of cationic uncouplers such as cyanine dyes and cupper $o$-phenanthroline was due to induction of PT pores. ${ }^{14)}$ As an extension of that study, we further characterized the actions of cyanine dyes on mitochondria in more detail in the present study. Both cyanine dyes tri-S- $\mathrm{C}_{4}(5)$ and tri-S- $\mathrm{C}_{7}(5)$ caused Pidependent acceleration of mitochondrial respiration like the well-known PT inducer $\mathrm{Ca}^{2+}$. However, different from the action of $\mathrm{Ca}^{2+}$, the action of cyanine dyes consisted of 2 phases: at lower concentrations, the acceleration of mitochondrial respiration was almost completely sensitive to CsA, but at higher concentrations, it was only slightly sensitive to the 
PT inhibitor. The CsA-sensitive action of cyanine dyes can be attributed to induction of the ordinary PT pore, but their CsA-insensitive action can not. Such a biphasic effect was also observed with the wasp venom peptide mastoparan. ${ }^{23)}$ Characterization of CsA-insensitive action of cyanine dyes, especially in the aspect of similarity/difference with that induced by mastoparan, is currently in progress.

We found that the cyanine dyes also induced the release of mitochondrial cytochrome $\mathrm{c}$. This effect was partially sensitive to CsA, as observed with their acceleration of the mitochondrial respiration. Based on these results, the following 2 conclusions can be drawn regarding the action of cyanine dyes. First, in the $\mathrm{Pi}$ containing medium $(+\mathrm{Pi})$, the degree of cytochrome c release was in good agreement with that of respiratory release. Second, not only CsA-sensitive but also CsA-insensitive action is effective for the release of mitochondrial cytochrome $\mathrm{c}$.

The requirement of phosphate for acceleration of mitochondrial respiration was more moderate than in the case of $\mathrm{Ca}^{2+}$. Namely, in the absence of Pi, $\mathrm{Ca}^{2+}$ did not show any effect on the respiration even at high concentrations such as $500 \mu \mathrm{M}$. On the contrary, cyanine dyes caused acceleration of mitochondrial respiration both with and without $\mathrm{Pi}$, although the induction of respiratory release in the absence of Pi required higher concentrations of cyanine dyes than that with Pi and its magnitude was smaller. The accelerating effect of cyanine dyes on the mitochondrial respiration in the absence of Pi seemed to be quite different from that in the presence of $\mathrm{Pi}$, since the former was not associated with the induction of mitochondrial swelling or release of mitochondrial cytochrome c. Possibly, in the absence of $\mathrm{Pi}$, cyanine dyes might perturb the inner mitochondrial membrane, making it permeable only to small ions such as $\mathrm{H}^{+}$. The status of the mitochondrial membrane treated with cyanine dye in the absence of $\mathrm{Pi}$ is being currently investigated further.

Numerous reports characterizing the mitochondrial PT have been published. However, explanation of the PT at the molecular level is still wanting. Thus, characterization of the PT induced under various conditions is very important. In the present study, cyanine dyes were found to show 3 effects on the mitochondrial structure and functions in a phosphatedependent manner. Thus, they are expected to be effective tools for better understanding of the molecular features of mitochondrial PT.

\section{REFERENCES}

1) Rottenberg, H. (1979) The measurement of membrane potential and delta $\mathrm{pH}$ in cells, organelles and vesicles. Methods Enzymol., 55, 547-569.

2) Waggoner, A. S. (1979) The use of cyanine dyes for the determination of membrane potentials in cells, organelles and vesicles. Methods Enzymol., 55, 689_ 695.

3) Terada, H., Nagamune, H., Osaki, Y. and Yoshikawa, K. (1981) Specific requirement for inorganic phosphate for induction of bilayer membrane conductance by the cationic uncoupler carbocyanine dye. Biochim. Biophys. Acta, 646, 488-490.

4) Terada, H. and Nagamune, H. (1983) A cyanine dye tri-S- $\mathrm{C}_{7}(5)$. Phosphate-dependent cationic uncoupler of oxidative phosphorylation in mitochondria. Biochim. Biophys. Acta, 723, 7-15.

5) Terada, H., Nagamune, H., Morikawa, N. and Ikuno, M. (1985) Uncoupling of oxidative phosphorylation by divalent cationic cyanine dye. Participation of phosphate transporter. Biochim. Biophys. Acta, 807, 168-176.

6) Takeguchi, N., Saitoh, T., Morii, M., Yoshikawa, K. and Terada, H. (1985) Formation of a leakage-type ion pathway in lipid bilayer membranes by divalent cationic cyanine dyes in cooperation with inorganic phosphate. Role of the cyanine dye in uncoupling of oxidative phosphorylation. J. Biol. Chem., 260 , 9158-9161.

7) Terada, H. (1981) The interaction of highly active uncouplers with mitochondria. Biochim. Biophys. Acta, 639, 225-242.

8) Terada, H. (1990) Uncouplers of oxidative phosphorylation. Environ. Health Perspect., 87, 213-218.

9) Skulachev, V. P. (1998) Uncoupling: new approaches to an old problem of bioenergetics. Biochim. Biophys. Acta, 1363, 100-124.

10) Shinohara, Y. and Terada, H. (2000) Uncouplers of oxidative phosphorylation in mitochondria. In Membrane Structure in Disease and Drug Therapy (Zimmer, G. D., Ed.), Marcel Dekker, New York, pp. 107-26.

11) Gunter, T. E. and Pfeiffer, D. R. (1990) Mechanisms by which mitochondria transport calcium. Am.J. Physiol., 258, C755-C786.

12) Zoratti, M. and Szabo, I. (1995) The mitochondrial permeability transition. Biochim. Biophys. Acta, 1241, 139-176.

13) Bernardi, P. (1999) Mitochondrial transport of cations: channels, exchangers, and permeability transition. Physiol. Rev., 79, 1127-1155.

14) Shinohara, Y., Bandou, S., Kora, S., Kitamura, S., Inazumi, S. and Terada, H. (1998) Cationic uncouplers of oxidative phosphorylation are induc- 
ers of mitochondrial permeability transition. FEBS Lett., 428, 89-92.

15) Fournier, N., Ducet, G. and Crevat, A. (1987) Action of cyclosporin on mitochondrial calcium fluxes. J. Bioenerg. Biomembr., 19, 297-303.

16) Broekemeier, K. M., Dempsey, M. E. and Pfeiffer, D. R. (1989) Cyclosporin A is a potent inhibitor of the inner membrane permeability transition in liver mitochondria. J. Biol. Chem., 264, 7826-7830.

17) Sokolove, P. M. and Kinnally, K. W. (1996) A mitochondrial signal peptide from Neurospora crassa increases the permeability of isolated rat liver mitochondria. Arch. Biochem. Biophys., 336, 69-76.

18) Sokolove, P. M. and Haley, L. M. (1996) Butylated hydroxytoluene and inorganic phosphate plus $\mathrm{Ca}^{2+}$ increase mitochondrial permeability via mutually exclusive mechanisms. J. Bioenerg. Biomembr., 28, 199-206.
19) Shinohara, Y., Almofti, M. R., Yamamoto, T., Ishida, T., Kita, F., Kanzaki, H., Ohnishi, M., Yamashita, K., Shimizu, S. and Terada, H. (2002) Permeability transition-independent release of mitochondrial cytochrome c induced by valinomycin. Eur. $J$. Biochem., 269, 5224-5230

20) Crompton, M. (1999) The mitochondrial permeability transition pore and its role in cell death. Biochem. $J ., 341,233-249$.

21) Kroemer, G. and Reed, J. C. (2000) Mitochondrial control of cell death. Nat. Med., 6, 513-519.

22) Bernardi, P., Petronilli, V., Di Lisa, F. and Forte, M. (2001) A mitochondrial perspective on cell death. Trends. Biochem. Sci., 26, 112-117.

23) Pfeiffer, D. R., Gudz, T. I., Novgorodov, S. A. and Erdahl, W. L. (1995) The peptide mastoparan is a potent facilitator of the mitochondrial permeability transition. J. Biol. Chem., 270, 4923-4932. 